\title{
DOMINANT STRATEGY IMPLEMENTATION with a Convex Product Space of Valuations
}

\section{by}

Katherine Cuff, Sunghoon Hong, Jesse Schwartz, Quan Wen, and John A. Weymark

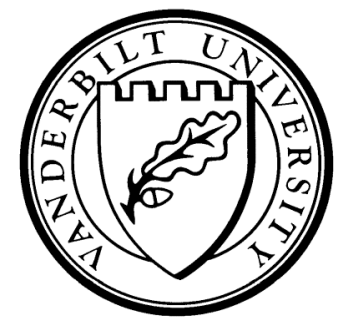

Working Paper No. 11-W04

May 2011

DEPARTMENT OF ECONOMICS

VANDERBILT UNIVERSITY

NASHVILLE, TN 37235

www.vanderbilt.edu/econ 


\title{
Dominant Strategy Implementation with a Convex Product Space of Valuations
}

Katherine Cuff ${ }^{1}$, Sunghoon Hong ${ }^{2}$, Jesse A. Schwartz ${ }^{3}$, Quan Wen ${ }^{4}$, and John A. Weymark ${ }^{5}$

1 Department of Economics, McMaster University, 1280 Main Street West, Hamilton, Ontario L8S 4M4, Canada.

E-mail: cuffk@mcmaster.ca

2 Department of Economics, Vanderbilt University, VU Station B \#35189, 2301 Vanderbilt Place, Nashville, TN 37215-1819, USA.

E-mail: sunghoon.hong@vanderbilt.edu

3 Department of Economics, Finance, and Quantitative Analysis, Kennesaw State University, 1000 Chastain Road, Box 0403, Kennesaw, GA 30144, USA.

E-mail: jschwar7@kennesaw.edu

${ }^{4}$ Department of Economics, Vanderbilt University, VU Station B \#35189, 2301 Vanderbilt Place, Nashville, TN 37235-1819, U.S.A.

E-mail: quan.wen@vanderbilt.edu

${ }^{5}$ Department of Economics, Vanderbilt University, VU Station B \#35189, 2301 Vanderbilt Place, Nashville, TN 37235-1819, U.S.A.

E-mail: john.weymark@vanderbilt.edu

May 2011.

\begin{abstract}
A necessary and sufficient condition for dominant strategy implementability when preferences are quasilinear is that, for any individual $i$ and any choice of the types of the other individuals, all $k$-cycles in $i$ 's allocation graph have nonnegative length for every integer $k \geq 2$. Saks and $\mathrm{Yu}$ (Proceedings of the 6th ACM Conference on Electronic Commerce (EC'05), 2005, 286-293) have shown that when the number of outcomes is finite and $i$ 's valuation type space is convex, nonnegativity of the length of all 2-cycles is sufficient for the nonnegativity of the length of all $k$-cycles. In this article, it is shown that if each individual's valuation type space is a convex product space and a mild domain regularity condition is satisfied, then (i) the nonnegativity of all 2-cycles implies that all $k$-cycles have zero length and (ii) all 2 -cycles having zero length is necessary and sufficient for dominant strategy implementability.
\end{abstract}

Keywords: 2-cycle condition, dominant strategy implementation, mechanism design, revenue equivalence, Rockafellar-Rochet Theorem, Saks-Yu Theorem.

JEL classification numbers: D44, D71, D82. 


\section{Introduction}

New insights into mechanism design theory, particularly when types are multidimensional, have recently been obtained using graph theory and linear programming. While the literature that uses these techniques focuses on obtaining general results that are not restricted to particular applications of the mechanism design framework, the results that have been obtained can be used in a wide variety of applications, such as auction design and the provision of public goods.

One issue that has attracted considerable attention is the development of necessary and sufficient conditions for dominant strategy implementability of an allocation function that chooses an outcome based on the reported type profile (a list of types, one for each individual) when the type space is restricted. Outcomes may be purely public or they may have private components. The starting point for this literature is a well-known necessary and sufficient condition for dominant strategy implementability for an arbitrary type space when utilities are quasilinear (linear in the payment) due to Rockafellar (1970) and Rochet (1987). Gui, Müller, and Vohra (2004) have provided a graph-theoretic interpretation of this condition: for any individual $i$ and any choice of the types of the other individuals, all cycles with a finite number of arcs in a directed graph defined using the valuations of the outcomes by individual $i$ have nonnegative length. In other words, for every integer $k \geq 2$, any cycle with $k$ arcs (a $k$-cycle) has nonnegative length.

It may be difficult to verify that this condition is satisfied if there are more than a few possible outcomes. To help overcome this problem, Bikhchandani, Chatterji, Lavi, Mu'alem, Nisan, and Sen (2006) have identified a fairly abstract domain richness condition for which it is sufficient for dominant strategy implementability that all 2-cycles have nonnegative length. Saks and Yu (2005) have shown that when there are a finite number of outcomes, if for any individual, the set of his possible valuations of these outcomes is convex for any choice of the types of the other individuals and if all 2-cycles in the corresponding graph have nonnegative length, then all cycles with an arbitrary number of arcs also have nonnegative length and, hence, the Rockafellar-Rochet sufficient condition for dominant strategy implementability is satisfied. Extensions and variants of Saks and Yu's results have been established by Archer and Kleinberg (2008), Ashlagi, Braverman, Hassidim, and Monderer (2010), and Berger, Müller, and Naeemi (2009, 2010).

In this article, we strengthen the assumption of Saks and Yu (2005) that the set of individual valuations is convex by requiring that it be a convex product space. With the addition of a mild regularity condition, we show that if all 2-cycles have nonnegative length when our domain restriction is satisfied, then in fact all cycles have zero length. In proving this result, we identify and exploit some geometric properties of this problem. An implication of this result is that all 2-cycles having zero length is necessary and sufficient for dominant strategy implementability given our assumptions. 
In order to state our results more precisely, we need to distinguish between the traditional concept of a type, here referred to as a characteristic type, and a valuation type, which we define below. We consider a direct mechanism that consists of an allocation function and a payment function. For each characteristic type profile, these functions determine an outcome and a payment (possibly negative) from each individual. The allocation function is dominant strategy implementable if there exists a payment function such that reporting one's true characteristic type is always a dominant strategy in the resulting direct mechanism.

We assume that the set of possible characteristic type profiles is the Cartesian product of the possible types for each individual and that for a fixed characteristic type, utility is quasilinear. For a given individual $i$ and given types of the other individuals, following Gui, Müller, and Vohra (2004), we define a complete directed graph called the characteristic graph whose nodes are the possible characteristic types of individual $i$ and the length (which could be negative) of the directed arc joining type $s^{i}$ to $t^{i}$ is the change in the valuation of the outcome obtained by individual $i$ when he is of type $t^{i}$ if he truthfully reports $t^{i}$ instead of $s^{i}$. Note that the payments are being ignored in this construction. In terms of characteristic graphs, the Rockafellar-Rochet Theorem shows that an allocation function is dominant strategy implementable if and only if for every individual $i$ and characteristic types of the other individuals, all $k$-cycles in the corresponding characteristic graph have nonnegative length for every integer $k \geq 2$.

When there are a finite number of outcomes, for a given individual $i$ and given characteristic types of the other individuals, we can equivalently describe $i$ 's characteristic type $t^{i}$ by the vector $v^{t^{i}}$ in $\mathbb{R}^{m}$ whose $j$ th component is the value of the $j$ th outcome when he is of type $t^{i}$. Here, $m$ is the number of outcomes that are attainable for the possible reported types of individual $i$ given the characteristic types of the other individuals. This vector $v^{t^{i}}$ is $i$ 's valuation type and the set of such types is $i$ 's valuation space (which depends on the types of the other individuals). Again following Gui, Müller, and Vohra (2004), this set of valuation types can be used to define a new graph, the allocation graph, whose nodes are the set of attainable outcomes and whose directed arc from $a$ to $b$ is the infimum of the change in valuation for $i$ of having $b$ instead of $a$ over all characteristic types for him for which the allocation function assigns $b$. The Rockafellar-Rochet Theorem can be restated in terms of the nonnegativity of all $k$-cycles in these allocation graphs.

The result of Saks and Yu (2005) stated informally above assumes that there are a finite number of outcomes and that, for each individual $i, i$ 's valuation type space is convex for any fixed types of the other individuals. With these assumptions, they show that it is sufficient for the nonnegativity of all $k$-cycles in $i$ 's allocation graph that every 2-cycle has nonnegative length. Thus, in view of the Rockafellar-Rochet Theorem, this 2-cycle nonnegativity condition is necessary and sufficient for dominant strategy implementability. This result is the Saks-Yu Theorem. 
We strengthen Saks and Yu's convexity assumption by requiring that, for any individual $i$ and for any fixed types of the other individuals, $i$ 's valuation type space is a convex product space; that is, it is the product of nondegenerate intervals of $\mathbb{R}$. We also suppose that $i$ 's valuation type space satisfies a regularity condition that ensures that there exists an open set of valuation types for $i$ that results in $a$ being chosen for each outcome $a$ that is attainable given the types of the other individuals. Our convex product set assumption and our regularity condition are satisfied if $i$ 's valuation type space is all of $\mathbb{R}^{m}$, that is, if it is unrestricted. ${ }^{1}$ With our assumptions, in our main theorem, we show that if all 2-cycles in $i$ 's allocation graph have nonnegative length, then all $k$-cycles in this graph have zero length for every integer $k \geq 2$. It then follows from this result and the Rockafellar-Rochet Theorem that an allocation function is dominant strategy implementable if and only if all 2cycles have zero length in every allocation graph, that is, in every allocation graph obtained by selecting an individual $i$ and fixing the types of the other individuals.

An allocation function that is dominant strategy implementable satisfies the revenue equivalence property if the payment functions that implement it have the property that for each individual $i$, given the types of the other individuals, the implementing payment functions for $i$ only differ by a constant. Revenue equivalence was first analyzed by Myerson (1981) in his study of the design of optimal auctions for a single good when the individual characterstic type spaces are one-dimensional. Heydenreich, Müller, Uetz, and Vohra (2009, Corollary 1) show that revenue equivalence is satisfied by an allocation function that is dominant strategy implementable if and only if all 2-cycles have zero length in every allocation graph. Thus, an implication of our main result is that, given our assumptions, our zero-length 2-cycle condition is not only necessary and sufficient for dominant strategy implementability, it is also necessary and sufficient for revenue equivalence.

In order to prove that all 2-cycles in i's allocation graph have nonnegative length, Saks and Yu (2005) use $i$ 's allocation graph to define a new graph with the same set of nodes for which the length of the directed arc from $a$ to $b$ is the total change in $i$ 's valuation along a particular kind of path in $i$ 's valuation type space. They show that under their assumptions, the length of any directed arc in this new graph bounds from below the length of this arc in $i$ 's allocation graph and that the length of any $k$-cycle in this new graph is zero, from which their theorem follows. In our proofs, we do not need to consider this auxillary graph.

\footnotetext{
${ }^{1}$ For a finite set of outcomes containing at least three outcomes, if all of the valuation type spaces are unrestricted, then Roberts (1979, Theorem 3.1) shows that an allocation function is dominant strategy implementable if and only if the outcome that is chosen when the individuals have characteristic types $\left(t^{1}, \ldots, t^{n}\right)$ is the outcome that maximizes the sum over individuals of an affine function of the individual valuations. For graph-theoretic proofs of Roberts' Theorem, see Lavi, Mu'alem, and Nisan (2009).
} 
Our main theorem generalizes one of the results in Lavi, Mu'alem, and Nisan (2009). They show that if $i$ 's valuation type space is unrestricted and if all 2-cycles in $i$ 's allocation graph have nonnegative length, then these 2cycles have zero length. We do not assume that $i$ 's valuation type space is unrestricted and we show that all $k$-cycles have zero length, not just the 2cycles. Lavi, Mu'alem, and Nisan (2009) also show that a necessary condition for dominant strategy implementability of the allocation function when $i$ 's valuation type space is unrestricted is that each 3-cycle in $i$ 's allocation graph has zero length, not just a nonnegative length, as required by the RockafellarRochet Theorem. This result is implied by combining our characterization theorem for dominant strategy implementability with our main theorem.

The plan of the rest of this article is as follows. In Section 2, we introduce the model and the characteristic and allocation graphs. We also present two versions of the Rockafellar-Rochet Theorem using these graphs. In Section 3, we consider the Saks-Yu Theorem. Next, in Section 4, we investigate the geometry of the partition of an individual's valuation type space into regions that are allocated the same outcome. In Section 5 , we state and prove our main theorem. We also provide some geometric intuition for our results. Finally, in Section 6 , we offer some concluding remarks.

\section{Dominant Strategy Implementability and the Rockafellar-Rochet Theorem}

Let $N$ be a finite set of $n$ individuals and $\Omega$ be a finite set of outcomes. For each $i \in N$, let $T^{i}$ denote the characteristic type space of individual $i$ with typical element $t^{i}$. For now, no assumptions are made about the structure of $T^{i}$. The value of $t^{i}$ is private information. Let $T^{-i}=\times_{j \in N \backslash\{i\}} T^{j}$ denote the characteristic type space of all individuals other than individual $i$. A characteristic type profile is written as $\left(t^{i}, t^{-i}\right) \in T^{i} \times T^{-i}$.

For each $i \in N$, let $v^{i}: \Omega \times T^{i} \rightarrow \mathbb{R}$ be the valuation function of individual $i$. This function assigns a value $v^{i}\left(a \mid t^{i}\right)$ to each outcome $a \in \Omega$ and characteristic type $t^{i} \in T^{i}$. Thus, an individual's valuation of an outcome only depends on his private characteristic type.

A direct mechanism $(G, P)$ consists of an allocation function $G: T^{i} \times$ $T^{-i} \rightarrow \Omega$ and a payment function $P: T^{i} \times T^{-i} \rightarrow \mathbb{R}^{n}$. The function $P$ may be written as $P=\left(P^{1}, \ldots, P^{n}\right)$, where $P^{i}$ is the payment function for individual $i$. For each type profile, $G$ determines an outcome in $\Omega$ and $P^{i}$ specifies a payment (which could be negative) from individual $i$.

An individual need not report his true type. Given the other individuals' reported types $t^{-i} \in T^{-i}$, the utility of individual $i$ with characteristic type $t^{i} \in T^{i}$ and reported type $s^{i} \in T^{i}$ is

$$
v^{i}\left(G\left(s^{i}, t^{-i}\right) \mid t^{i}\right)-P^{i}\left(s^{i}, t^{-i}\right) .
$$


Definition. An allocation function $G$ is dominant strategy implementable if there exists a payment function $P$ such that for all $i \in N$ and all $t^{-i} \in T^{-i}$,

$$
v^{i}\left(G\left(t^{i}, t^{-i}\right) \mid t^{i}\right)-P^{i}\left(t^{i}, t^{-i}\right) \geq v^{i}\left(G\left(s^{i}, t^{-i}\right) \mid t^{i}\right)-P^{i}\left(s^{i}, t^{-i}\right), \quad \forall s^{i}, t^{i} \in T^{i} .
$$

In other words, an allocation function is dominant strategy implementable if there exists a payment function for which each individual is at least as well off reporting his true type than reporting any other type regardless of what the other individuals report. As a consequence, in analyzing dominant strategy implementability, without loss of generality, we can consider a fixed individual $i \in N$ and fixed types $t^{-i} \in T^{-i}$ of the other individuals in the subsequent discussion. Henceforth, we let $t=t^{i}$ and $T=T^{i}$.

For the given choice of $t^{-i}$, let $A=\left\{a_{1}, \ldots, a_{m}\right\}$ be the finite set of $m$ attainable outcomes for the allocation function $G$. That is,

$$
A=\left\{a \in \Omega \mid G\left(t, t^{-i}\right)=a \text { for some } t \in T\right\} .
$$

By fixing $i \in N$ and $t^{-i} \in T^{-i},(G, P)$ defines a single person mechanism $(g, p)$ with allocation function $g: T \rightarrow A$ and payment function $p: T \rightarrow \mathbb{R}$ obtained by setting

$$
g(t)=G\left(t, t^{-i}\right) \text { and } p(t)=P^{i}\left(t, t^{-i}\right), \forall t \in T .
$$

Note that $g$ is surjective.

For all $a \in A$, let

$$
R_{a}=\{t \in T \mid g(t)=a\}
$$

be the set of characteristic types for $i$ that induce outcome $a$ using the allocation function $g$. By construction, $R_{a}$ is nonempty for all $a \in A$. Because $g$ depends on the other individuals' types, $R_{a}$ also depends on their types.

For the mechanism $(g, p)$, the dominant strategy implementability condition (1) simplifies to

$$
v(g(t) \mid t)-p(t) \geq v(g(s) \mid t)-p(s), \quad \forall s, t \in T .
$$

For the allocation function $g$, the characteristic graph $T_{g}$ is the complete directed graph with nodes $T$ and arc length

$$
d(s, t)=v(g(t) \mid t)-v(g(s) \mid t)
$$

for the directed arc $(s, t)$ from $s$ to $t^{2}$ That is, $d(s, t)$ is the change in the valuation if the true characteristic type $t$ is reported instead of the characteristic type $s$. This change in valuation is not the overall change in utility because the payments have not been taken into account.

${ }^{2}$ We exclude loops. That is, there are no arcs from a node to itself. The characteristic graph, as well as the allocation graph defined below, were introduced by Gui, Müller, and Vohra (2004). We adopt their terminology in calling (3) a "length" instead of an edge weight even though it may be negative. 
For any integer $k \geq 2$, a $k$-cycle in the characteristic graph $T_{g}$ is a sequence of distinct $\operatorname{arcs}\left(t_{1}, t_{2}\right), \ldots,\left(t_{k-1}, t_{k}\right),\left(t_{k}, t_{1}\right)$ whose length is defined to be the sum of the lengths of the arcs in the cycle. That is, the length of the $k$-cycle is $d\left(t_{1}, t_{2}\right)+\cdots+d\left(t_{k-1}, t_{k}\right)+d\left(t_{k}, t_{1}\right){ }^{3}$

Rochet (1987, Theorem 1) uses a theorem about subdifferentials of multidimensional convex functions due to Rockafellar (1970, Theorem 24.8) to provide necessary and sufficient conditions for an allocation function to be dominant strategy implementable. This result is known as the RockafellarRochet Theorem. Theorem 1 provides a statement of this theorem in terms of the characteristic graph $T_{g}$.

Theorem 1. The allocation function $g: T \rightarrow A$ is dominant strategy implementable if and only if for every integer $k \geq 2$, any $k$-cycle in the characteristic graph $T_{g}$ has nonnegative length.

If $g$ is dominant strategy implementable and $g(s)=g(t)$, then $p(s)=$ $p(t)$ as well. As a consequence, dominant strategy implementability of $g$ is equivalent to the existence of a payment schedule $\rho: A \rightarrow \mathbb{R}$ that assigns payments to outcomes for which

$$
g(t) \in \arg \max _{a \in A} v(a \mid t)-\rho(a), \quad \forall t \in T .
$$

That is, given the payment schedule $\rho, g(t)$ is an outcome that maximizes the utility of a type $t$ individual. This equivalence result is known as the taxation principle (see, e.g., Rochet, 1987) because it was originally obtained in the context of optimal tax theory.

Given the allocation function $g$, the corresponding allocation graph $\Gamma_{g}$ is the complete directed graph that has $A$ as the set of nodes and $\ell(a, b)$ as the length of the directed arc from node $a$ to node $b$, where

$$
\ell(a, b)=\inf _{t \in R_{b}}[v(b \mid t)-v(a \mid t)]=\inf _{t \in R_{b}}[v(g(t) \mid t)-v(a \mid t)], \quad \forall a, b \in A(a \neq b) .^{4}
$$

In this graph, the length (which could be negative) of the directed arc from $a$ to $b$ is the infimum of the change in valuation of having $b$ instead of $a$ over the set of all characteristic types for which the outcome function assigns $b$.

For any two nodes $a$ and $b$ in the allocation graph $\Gamma_{g}$, a path from $a$ to $b$ is a sequence of distinct $\operatorname{arcs}\left(a_{1}, a_{2}\right), \ldots,\left(a_{k-1}, a_{k}\right)$ for which $a=a_{1}$ and $b=a_{k}$. For any integer $k \geq 2$, a $k$-cycle in the allocation graph $\Gamma_{g}$ is a path with $k$ arcs whose endpoints are both the same. That is, it is a sequence of $\operatorname{arcs}\left(a_{1}, a_{2}\right), \ldots,\left(a_{k-1}, a_{k}\right),\left(a_{k}, a_{1}\right)$. The length of a path or $k$-cycle is the sum of the lengths of the arcs that comprise it.

\footnotetext{
${ }^{3}$ Note that because there are no loops, there are no 1-cycles.

${ }^{4}$ We adopt the convention that the infimum and supremum are equal to $-\infty$ and $\infty$, respectively, when they are not finite. Our assumptions do not rule out the possibility that $\ell(a, b)=-\infty$. As shown by Mishra (2009) and Vohra (2011), $\ell(a, b)$ is finite for all $a, b \in A$ if $(2)$ is satisfied.
} 
The Rockafellar-Rochet Theorem can be restated using allocation graphs. ${ }^{5}$

Theorem 2. The allocation function $g: T \rightarrow A$ is dominant strategy implementable if and only if for every integer $k \geq 2$, any $k$-cycle in the allocation graph $\Gamma_{g}$ has nonnegative length.

Proof. First, suppose that for every integer $k \geq 2$, any $k$-cycle in $\Gamma_{g}$ has nonnegative length. Because there are a finite number of outcomes in $A$ and, hence, a finite number of nodes in $\Gamma_{g}$, between any two nodes in $A$ there exists a shortest path. Fix $a \in A$. Let $\bar{p}: T \rightarrow \mathbb{R}$ be the length of a shortest path from $a$ to $g(t)$. We shall show that the mechanism $(g, \bar{p})$ satisfies the dominant strategy implementability condition (2).

Consider any two types $s, t \in T$. We have

$$
\bar{p}(t) \leq \bar{p}(s)+\ell(g(s), g(t))
$$

because the length of a shortest path from $a$ to $g(t)$ cannot exceed the length of a path from $a$ to $g(s)$ to $g(t)$. It follows that

$$
\begin{aligned}
\bar{p}(t)-\bar{p}(s) & \leq \ell(g(s), g(t)) \\
& =\inf _{r \in R_{g(t)}}[v(g(t) \mid r)-v(g(s) \mid r)] \\
& \leq v(g(t) \mid t)-v(g(s) \mid t),
\end{aligned}
$$

thereby establishing (2).

Second, suppose that there exists a payment function $p: T \rightarrow A$ such that $(g, p)$ satisfies (2). Rearranging (2), we obtain

$$
p(t)-p(s) \leq v(g(t) \mid t)-v(g(s) \mid t), \quad \forall s, t \in T .
$$

Consider any $k$-cycle $\left(a_{1}, a_{2}\right), \ldots,\left(a_{k-1}, a_{k}\right),\left(a_{k}, a_{1}\right)$ for an arbitrary integer $k \geq 2$. Let $a_{k+1}=a_{1}$. Then, for all $\varepsilon>0$ and all $j=\{1, \ldots, k\}$, there exist $s^{\varepsilon, j} \in R_{a_{j}}$ such that

$$
\begin{aligned}
\sum_{j=1}^{k} \ell\left(a_{j}, a_{j+1}\right) & =\sum_{j=1}^{k} \inf _{t \in R_{a_{j+1}}}\left[v\left(a_{j+1} \mid t\right)-v\left(a_{j} \mid t\right)\right] \\
& >\sum_{j=1}^{k}\left[v\left(a_{j+1} \mid s^{\varepsilon, j+1}\right)-v\left(a_{j} \mid s^{\varepsilon, j+1}\right)-\varepsilon\right] .
\end{aligned}
$$

Noting that $s^{\varepsilon, k+1}=s^{\varepsilon, 1}$, it follows from (5) that

$$
\sum_{j=1}^{k}\left[v\left(a_{j+1} \mid s^{\varepsilon, j+1}\right)-v\left(a_{j} \mid s^{\varepsilon, j+1}\right)-\varepsilon\right] \geq \sum_{j=1}^{k}\left[p\left(s^{\varepsilon, j+1}\right)-p\left(s^{\varepsilon, j}\right)-\varepsilon\right] .
$$

5 This version of the Rockafellar-Rochet Theorem is stated without proof in Vohra (2011). A somewhat different proof may be found in Mishra (2009, Theorem 2). 
The sum on the right-hand side of (7) is $-k \varepsilon$. Hence, (6) and (7) imply that

$$
\sum_{j=1}^{k} \ell\left(a_{j}, a_{j+1}\right)>-k \varepsilon .
$$

Taking the limit as $\varepsilon$ goes to 0 in (8) shows that the length of this $k$-cycle is nonnegative.

\section{The Saks-Yu Theorem}

It may be computationally onerous to check that every cycle in either the characteristic graph $T_{g}$ or in the allocation graph $\Gamma_{g}$ has nonnegative length in order to determine if the allocation function $g$ is dominant strategy implementable. It follows from the Rockafellar-Rochet Theorem that a necessary condition for dominant strategy implementability is that all the 2-cycles have nonnegative length. Bikhchandani, Chatterji, Lavi, Mu'alem, Nisan, and Sen (2006) and Saks and Yu (2005) have identified alternative restrictions on $v$ under which this 2-cycle nonnegativity condition is also sufficient for dominant strategy implementability. Our results build on those of Saks and Yu.

The 2-cycle nonnegativity condition can be defined in either of our two graphs. Below we shall show that these two definitions are equivalent. We begin with the characteristic graph.

Definition. An allocation function $g$ satisfies the characteristic graph 2-cycle nonnegativity condition if

$$
d(s, t)+d(t, s) \geq 0, \quad \forall s, t \in T .
$$

It follows from (3) that (9) is equivalent to

$$
v(g(t) \mid t)-v(g(s) \mid t) \geq v(g(t) \mid s)-v(g(s) \mid s), \quad \forall s, t \in T .
$$

That is, the change in valuation obtained by replacing $g(s)$ with $g(t)$ is at least as large for type $t$ as for type $s$. For this reason, Bikhchandani, Chatterji, Lavi, Mu'alem, Nisan, and Sen (2006) call this condition weak monotonicity.

We now define the corresponding condition using the allocation graph.

Definition. An allocation function $g$ satisfies the allocation graph 2-cycle nonnegativity condition if

$$
\ell(a, b)+\ell(b, a) \geq 0, \quad \forall a, b \in A .
$$

Theorem 3 shows that these two 2-cycle nonnegativity conditions are equivalent. In light of this equivalence, we shall simply refer to this condition as the 2-cycle nonnegativity condition. 
Theorem 3. An allocation function $g: T \rightarrow A$ satisfies the characteristic graph 2-cycle nonnegativity condition if and only if it satisfies the allocation graph 2-cycle nonnegativity condition.

Proof. First, suppose the allocation rule $g$ satisfies the characteristic graph 2-cycle nonnegativity condition (9) but that, by way of contradiction, there exist outcomes $\hat{a}$ and $\hat{b}$ in $A$ such that $\ell(\hat{a}, \hat{b})+\ell(\hat{b}, \hat{a})<0$. Using the definition of $\ell$ in (4), we can rewrite the last inequality as

$$
\inf _{t \in R_{\hat{b}}}[v(\hat{b} \mid t)-v(\hat{a} \mid t)]+\inf _{s \in R_{\hat{a}}}[v(\hat{a} \mid s)-v(\hat{b} \mid s)]<0 .
$$

Thus, there exist characteristic types $\hat{s} \in R_{\hat{a}}$ and $\hat{t} \in R_{\hat{b}}$ such that

$$
[v(\hat{b} \mid \hat{t})-v(\hat{a} \mid \hat{t})]+[v(\hat{a} \mid \hat{s})-v(\hat{b} \mid \hat{s})]<0 .
$$

This inequality, however, contradicts (10), which is equivalent to the characteristic graph 2-cycle nonnegativity condition (9).

Second, suppose the allocation rule $g$ satisfies the allocation graph 2-cycle nonnegativity condition (11) but that, by way of contradiction, there exist types $\hat{s}$ and $\hat{t}$ in $T$ such that $d(\hat{s}, \hat{t})+d(\hat{t}, \hat{s})<0$ or, equivalently, that

$$
[v(\hat{b} \mid \hat{t})-v(\hat{a} \mid \hat{t})]+[v(\hat{a} \mid \hat{s})-v(\hat{b} \mid \hat{s})]<0,
$$

where $\hat{a}=g(\hat{s})$ and $\hat{b}=g(\hat{t})$. From this last inequality, it follows that

$$
\ell(\hat{a}, \hat{b})+\ell(\hat{b}, \hat{a})=\inf _{t \in R_{\hat{b}}}[v(\hat{b} \mid t)-v(\hat{a} \mid t)]+\inf _{s \in R_{\hat{a}}}[v(\hat{a} \mid s)-v(\hat{b} \mid s)]<0,
$$

which contradicts the allocation graph 2-cycle nonnegativity condition (11).

Each characteristic type $t \in T$ has associated with it a corresponding valuation type $v^{t}=\left(v_{a_{1}}^{t}, \ldots, v_{a_{m}}^{t}\right) \in \mathbb{R}^{m}$, where $v_{a}^{t}=v(a \mid t)$ for all $a \in A$. The $j$ th component of $v^{t}$ is the value of outcome $a_{j}$ when individual $i$ is of characteristic type $t$ given the fixed characteristic type profile $t^{-i}$ of the other individuals. Individual $i$ 's valuation type space (given $t^{-i}$ ) is

$$
\mathcal{V}=\left\{v^{t} \in \mathbb{R}^{m} \mid t \in T\right\} .
$$

If characteristic types $s$ and $t$ have the same associated valuation type $v$, there is then no loss of generality in identifying them (i.e., treating them as being the same characteristic type). Henceforth, we assume that if $s \neq t$, then $v^{s} \neq v^{t}$. With this assumption, there is a unique $t \in T$ associated with each $v \in \mathcal{V}$. Hence, individual $i$ can be equivalently characterized by his characteristic type $t$ or his valuation type $v$. Let $t^{v}$ denote the characteristic type associated with $v$. 
Saks and $\mathrm{Yu}(2005$, Theorem 4) show that if the valuation type space $\mathcal{V}$ is convex, then all $k$-cycles in the allocation graph $\Gamma_{g}$ have nonnegative length if $\Gamma_{g}$ satisfies the allocation graph 2-cycle nonnegativity condition (11). In view of the equivalence in Theorem 3, we thus have that the 2-cycle nonnegativity condition is sufficient for dominant strategy implementability when $\mathcal{V}$ is convex.

Theorem 4. If $\mathcal{V}$ is convex, then the allocation function $g: T \rightarrow A$ is dominant strategy implementable if the 2-cycle nonnegativity condition is satisfied.

As Saks and Yu have noted, it immediately follows from Theorem 4 and the Rockafellar-Rochet Theorem that the 2-cycle nonnegativity condition is necessary and sufficient for dominant strategy implementability when $\mathcal{V}$ is convex. This result, which is Theorem 1 in Saks and Yu (2005), is the SaksYu Theorem.

Theorem 5. If $\mathcal{V}$ is convex, then the allocation function $g: T \rightarrow A$ is dominant strategy implementable if and only if the 2-cycle nonnegativity condition is satisfied.

Note that the assumption that $\mathcal{V}$ is convex implicitly places restrictions on the characteristic type space $T$. In particular, $T$ cannot be discrete.

\section{Partitioning the Valuation Type Space}

Recall that $R_{a}$ is the set of characteristic types that the allocation function $g$ maps into outcome $a$. Because there is a bijection between the characteristic type space $T$ and the valuation type space $\mathcal{V}$, the sets $R_{a}$ for $a \in A$ induce a partition of $\mathcal{V}$ with each cell in the partition associated with the outcome assigned to valuation types in that cell. Our results are obtained by investigating the geometry of this partition.

The valuation type space $\mathcal{V}$ is a subset of $\mathbb{R}^{m}$. We first define some sets on all of $\mathbb{R}^{m}$ and then later restrict them to $\mathcal{V}$.

For all $a, b \in A$ with $a \neq b$, the difference set for $(a, b)$ is

$$
Q_{a b}=\left\{v \in \mathbb{R}^{m} \mid v_{a}-v_{b} \geq \ell(b, a)\right\} .
$$

$Q_{a b}$ is a closed halfspace. A valuation type $v$ is in $Q_{a b}$ if the change in valuation for individual $i$ of having object $a$ instead of $b$ is at least as large as the infimum of the change in valuation of having $b$ instead of $a$ over the set of all characteristic types for which the outcome function assigns $a$.

Difference sets are illustrated in Figure 1 for the case in which $A=\left\{a_{1}, a_{2}\right\}$. In this case, the two difference sets are

$$
Q_{a_{1} a_{2}}=\left\{v \in \mathbb{R}^{2} \mid v_{a_{2}} \leq-\ell\left(a_{2}, a_{1}\right)+v_{a_{1}}\right\}
$$




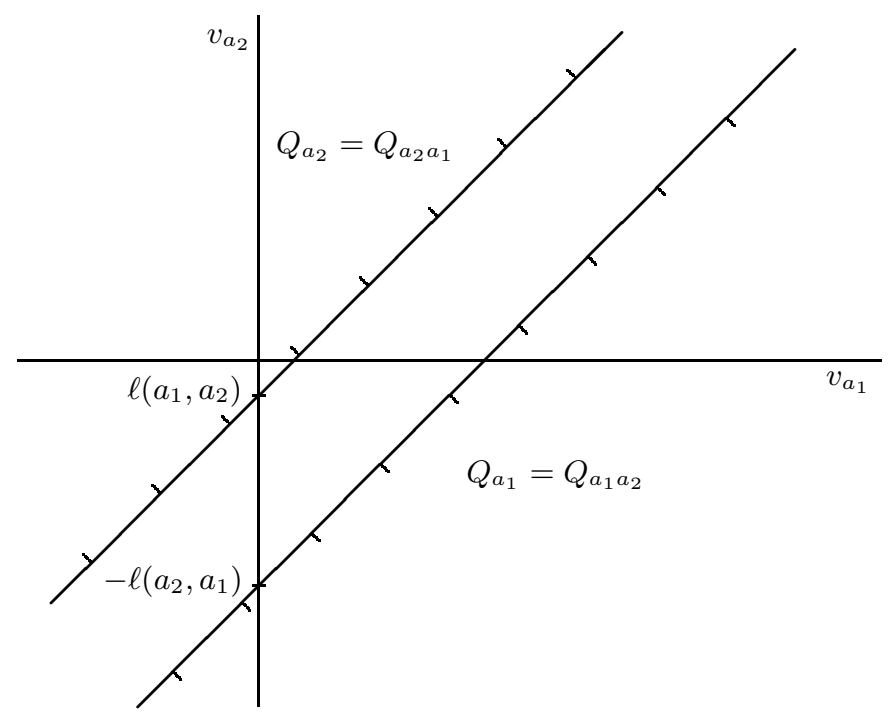

Fig. 1. Difference sets when $m=2$.

and

$$
Q_{a_{2} a_{1}}=\left\{v \in \mathbb{R}^{2} \mid v_{a_{2}} \geq \ell\left(a_{1}, a_{2}\right)+v_{a_{1}}\right\} .
$$

The boundaries of these two sets have slope equal to 1 and their vertical intercepts are $-\ell\left(a_{2}, a_{1}\right)$ and $\ell\left(a_{1}, a_{2}\right)$, respectively. The 2 -cycle nonnegativity condition $\ell\left(a_{1}, a_{2}\right)+\ell\left(a_{2}, a_{1}\right) \geq 0$ holds if and only $\ell\left(a_{1}, a_{2}\right) \geq-\ell\left(a_{2}, a_{1}\right)$. Hence, if the 2-cycle nonnegativity condition is satisfied, $Q_{a_{2} a_{1}}$ lies above $Q_{a_{1} a_{2}}$ and the interiors of these sets have an empty intersection.

For each $a \in A$, the difference set for $a$ is

$$
Q_{a}=\bigcap_{b \in A \backslash\{a\}} Q_{a b}
$$

$Q_{a}$ is the closed convex polyhedron obtained by intersecting the halfspaces $Q_{a b}$ for all outcomes $b$ distinct from $a$. For the two-outcome case illustrated in Figure $1, Q_{a_{1}}=Q_{a_{1} a_{2}}$ and $Q_{a_{2}}=Q_{a_{2} a_{1}}$.

For the case in which $A=\left\{a_{1}, a_{2}, a_{3}\right\}$, cross sections of the difference sets $Q_{a_{1}}, Q_{a_{2}}$, and $Q_{a_{3}}$ for a fixed valuation $\bar{v}_{a_{3}}$ of the third outcome are illustrated in Figure 2. Let

$$
\Upsilon=\left\{v \in \mathbb{R}^{3} \mid v_{a_{3}}=\bar{v}_{a_{3}}\right\} .
$$

In this diagram, $Q_{a_{1}} \cap \Upsilon$ is the intersection of

$$
Q_{a_{1} a_{2}} \cap \Upsilon=\left\{v \in \Upsilon \mid v_{a_{2}} \leq-\ell\left(a_{2}, a_{1}\right)+v_{a_{1}}\right\}
$$

and 


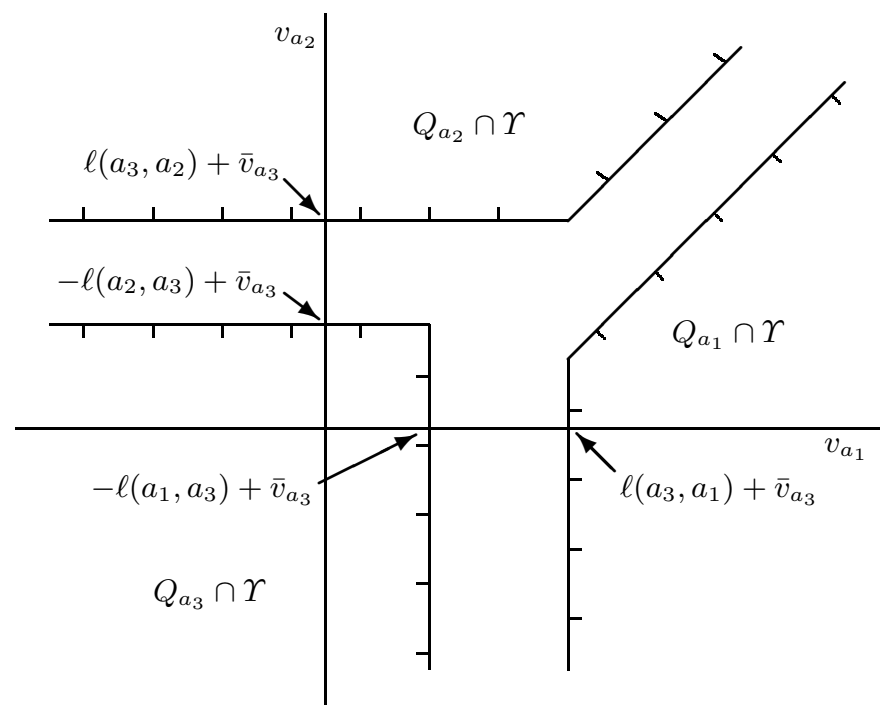

Fig. 2. Difference sets when $m=3$.

$$
Q_{a_{1} a_{3}} \cap \Upsilon=\left\{v \in \Upsilon \mid v_{a_{1}} \geq \ell\left(a_{3}, a_{1}\right)+\bar{v}_{a_{3}}\right\},
$$

$Q_{a_{2}} \cap \Upsilon$ is the intersection of

$$
Q_{a_{2} a_{1}} \cap \Upsilon=\left\{v \in \Upsilon \mid v_{a_{2}} \geq \ell\left(a_{1}, a_{2}\right)+v_{a_{1}}\right\}
$$

and

$$
Q_{a_{2} a_{3}} \cap \Upsilon=\left\{v \in \Upsilon \mid v_{a_{2}} \geq \ell\left(a_{3}, a_{2}\right)+\bar{v}_{a_{3}}\right\}
$$

and $Q_{a_{3}} \cap \Upsilon$ is the intersection of

$$
Q_{a_{3} a_{1}} \cap \Upsilon=\left\{v \in \Upsilon \mid v_{a_{1}} \leq-\ell\left(a_{1}, a_{3}\right)+\bar{v}_{a_{3}}\right\}
$$

and

$$
Q_{a_{3} a_{2}} \cap \Upsilon=\left\{v \in \Upsilon \mid v_{a_{2}} \leq-\ell\left(a_{2}, a_{3}\right)+\bar{v}_{a_{3}}\right\} .
$$

Let $\partial S$ denote the boundary of set $S$. The intersections of $Q_{a_{1} a_{2}}$ and $Q_{a_{2} a_{1}}$ with $\Upsilon$ do not depend on the choice of $\bar{v}_{a_{3}}$. Hence, for all $\bar{v}_{a_{3}}$, the upward sloping parts of $\partial Q_{a_{1}} \cap \Upsilon$ and $\partial Q_{a_{2}} \cap \Upsilon$ have slope equal to 1 and are contained in lines whose intercepts with the axes do not depend on $\bar{v}_{a_{3}}$. As $\bar{v}_{a_{3}}$ increases, the horizontal parts of $\partial Q_{a_{2}} \cap \Upsilon$ and $\partial Q_{a_{3}} \cap \Upsilon$ move up and the vertical parts of $\partial Q_{a_{1}} \cap \Upsilon$ and $\partial Q_{a_{3}} \cap \Upsilon$ move to the right.

When there are more than three outcomes, the analogue of Figure 2 is obtained by setting $\Upsilon=\left\{v \in \mathbb{R}^{m} \mid\left(v_{a_{3}}, \ldots, v_{a_{m}}\right)=\left(\bar{v}_{a_{3}}, \ldots, \bar{v}_{a_{m}}\right)\right\}$. In this case, the restrictions of the difference sets $Q_{a_{1}}$ and $Q_{a_{2}}$ to $\Upsilon$ have the same shapes as shown in Figure 2. Provided that the 2-cycle nonnegativity condition is satisfied, there is a single outcome $a_{d^{*}}$ that maximizes both $\ell\left(a_{d}, a_{1}\right)+$ 
$\bar{v}_{a_{d}}$ and $\ell\left(a_{d}, a_{2}\right)+\bar{v}_{a_{d}}$ for $d \neq 1,2$. The points in the vertical boundary of $Q_{a_{1}} \cap \Upsilon$ all have first coordinate equal to $\ell\left(a_{d^{*}}, a_{1}\right)+\bar{v}_{a_{d^{*}}}$ and the points in the horizontal boundary of $Q_{a_{2}} \cap \Upsilon$ all have second coordinate equal to $\ell\left(a_{d^{*}}, a_{2}\right)+\bar{v}_{a_{d^{*}}}$. Generically, the only other difference set that has a nonempty intersection with $\Upsilon$ is $Q_{a_{d^{*}}}$. It has the same shape as $Q_{a_{3}} \cap \Upsilon$ in Figure 2, with points in its vertical boundary having first coordinate equal to $-\ell\left(a_{1}, a_{d^{*}}\right)+$ $\bar{v}_{a_{d^{*}}}$ and points in its horizontal boundary having second coordinate equal to $-\ell\left(a_{2}, a_{d^{*}}\right)+\bar{v}_{a_{d^{*}}}$. It is, however, possible that there is an $a_{\bar{d}} \neq a_{d^{*}}$ for which $Q_{a_{d^{*}}} \cap \Upsilon=Q_{a_{\bar{d}}} \cap \Upsilon$. This happens when these sets are common boundary points of $Q_{a_{d^{*}}}$ and $Q_{a_{\bar{d}}}$.

There is a close connection between the set of characteristic types $T_{a}$ that are allocated outcome $a$ and the set $Q_{a} \cap \mathcal{V}$. Except for possibly some of the boundary points of $Q_{a}$, the set of characteristic types associated with valuation types in $Q_{a} \cap \mathcal{V}$ is $R_{a}$. More precisely, for any $t \in R_{a}$, the valuation type $v^{t}$ is in $Q_{a} \cap \mathcal{V}$. Moreover, if the allocation function satisfies the 2-cycle nonnegativity condition, then for any $v \in \mathcal{V}$ that is in the interior $Q_{a}^{\circ}$ of $Q_{a}$, the characteristic type $t^{v}$ is in $R_{a}$. Proofs of these results may be found in Mishra (2009) and Vohra (2011), but for completeness, we include them here.

Theorem 6. For any allocation function $g: T \rightarrow A$ and any outcome $a \in A$, (i) for any characteristic type $t \in R_{a}$, the valuation type $v^{t}$ is in $Q_{a} \cap \mathcal{V}$ and (ii) if $g$ satisfies the 2-cycle nonnegativity condition, then for any valuation type $v \in Q_{a}^{\circ} \cap \mathcal{V}$, the characteristic type $t^{v}$ is in $R_{a}$.

Proof. (i) By definition, $g(t)=a$ for any characteristic type $t \in R_{a}$. Therefore,

$$
v(a \mid t)-v(b \mid t) \geq \inf _{t \in R_{a}}[v(a \mid t)-v(b \mid t)]=\ell(b, a), \quad \forall b \in A \backslash\{a\} .
$$

Hence, by the definition of $Q_{a}$, we have $v^{t} \in Q_{a} \cap \mathcal{V}$.

(ii) Consider any valuation type $v \in Q_{a}^{\circ} \cap \mathcal{V}$. Because $v \in Q_{a}^{\circ}$, for the characteristic type $t^{v}$, we have

$$
v_{a}-v_{b}=v\left(a \mid t^{v}\right)-v\left(b \mid t^{v}\right)>\ell(b, a), \quad \forall b \in A \backslash\{a\} .
$$

Because the allocation rule $g$ satisfies the 2-cycle nonnegativity condition, $\ell(a, b) \geq-\ell(b, a)$ for all $b \in A \backslash\{a\}$. The last two inequalities then imply that

$$
v_{b}-v_{a}=v\left(b \mid t^{v}\right)-v\left(a \mid t^{v}\right)<-\ell(b, a) \leq \ell(a, b), \quad \forall b \in A \backslash\{a\} .
$$

Hence, $v \notin Q_{b} \cap \mathcal{V}$ for any $b \in A \backslash\{a\}$. Therefore, from part (i) it follows that $t^{v} \notin R_{b}$ for any $b \in A \backslash\{a\}$. Consequently, $t^{v}$ must be in $R_{a}$.

An immediate implication of Theorem 6 is that for all $a, b \in A, Q_{a}^{\circ} \cap$ $Q_{b}^{\circ}=\varnothing$ if the 2-cycle nonnegativity condition is satisfied. Furthermore, if $v \in Q_{a} \cap Q_{b} \cap \mathcal{V}$, then $v_{a}-v_{b}=\ell(b, a)=-\ell(a, b) .{ }^{6}$

\footnotetext{
${ }^{6}$ See Saks and Yu (2005, Proposition 5).
} 
Our next theorem shows that the allocation function $g$ satisfies a monotonicity property when the 2-cycle nonnegativity condition is satisfied. Specifically, if the valuation of the chosen outcome, say $a$, increases and the valuation of no other outcome decreases, then no outcome $b$ different from $a$ can be chosen unless $b$ 's valuation also increases.

Theorem 7. If the allocation function $g: T \rightarrow A$ satisfies the 2-cycle nonnegativity condition, then for any characteristic type $t \in R_{a}$ and any valuation type $v^{\prime} \in \mathcal{V}$ with $v^{\prime} \geq v^{t}$ for which $v_{a}^{\prime}>v_{a}^{t}$ and $v_{b}^{\prime}=v_{b}^{t}$, the characteristic type $t^{v^{\prime}}$ is not in $R_{b}$.

Proof. Consider any $a \in A$ and $t \in R_{a}$. By Theorem 6, we have

$$
v_{a}^{t}-v_{c}^{t} \geq \ell(c, a), \quad \forall c \in A \backslash\{a\} .
$$

Consider any $b \in A \backslash\{a\}$ and any valuation type $v^{\prime} \in \mathcal{V}$ with $v^{\prime} \geq v^{t}$ for which $v_{a}^{\prime}>v_{a}^{t}$ and $v_{b}^{\prime}=v_{b}^{t}$. The preceding inequality then implies that

$$
v_{b}^{\prime}-v_{a}^{\prime}<v_{b}^{t}-v_{a}^{t} \leq-\ell(b, a) .
$$

By the 2-cycle nonnegativity condition, we thus have

$$
v_{b}^{\prime}-v_{a}^{\prime}<\ell(a, b) .
$$

Hence, by Theorem $6, t^{v^{\prime}} \notin R_{b}$.

\section{The Main Theorem}

In this section, we replace the Saks-Yu assumption that the valuation type space $\mathcal{V}$ is convex with the more restrictive assumption that it is the product of nondegenerate intervals, what we call a convex product space.

Definition. The valuation type space $\mathcal{V}$ is a convex product space if

$$
\mathcal{V}=\times_{a \in A}\left\langle L_{a}, U_{a}\right\rangle,
$$

where for all $a \in A,\left\langle L_{a}, U_{a}\right\rangle$ is any type of interval of $\mathbb{R}$ with endpoints $L_{a}$ and $U_{a}$ for which $L_{a}<U_{a}{ }^{7}$

By construction, $Q_{a} \cap \mathcal{V} \neq \varnothing$ for all $a \in A$. We henceforth assume that $Q_{a} \cap \mathcal{V}$ has a nonempty interior for all $a \in A$. We refer to this restriction as the interiority assumption. This condition necessarily holds if $\mathcal{V}$ is open, which is the case if, for example, $\mathcal{V}=\mathbb{R}^{m}$. If $\mathcal{V}$ is not open, requiring $Q_{a} \cap \mathcal{V}$ to have a nonempty interior is a mild regularity condition.

\footnotetext{
${ }^{7}$ We permit $L_{a}$ to be $-\infty$ and $U_{a}$ to be $\infty$.
} 
As we have noted, Saks and Yu (2005) show that if an allocation function $g: T \rightarrow A$ satisfies the 2-cycle nonnegativity condition and $\mathcal{V}$ is convex, then all $k$-cycles in the allocation graph $\Gamma_{g}$ have nonnegative length. Our main theorem shows that, in fact, all of these $k$-cycles have zero length if we additionally assume that $\mathcal{V}$ is a convex product space and the intersection of each difference set $Q_{a}$ with $\mathcal{V}$ has a nonempty interior.

Theorem 8. If $(i)$ the allocation function $g: T \rightarrow A$ satisfies the 2-cycle nonnegativity condition, $(i i)$ the valuation type space $\mathcal{V}$ is a convex product space, and (iii) $Q_{a} \cap \mathcal{V}$ has a nonempty interior for all $a \in A$, then for every integer $k \geq 2$, any $k$-cycle in the allocation graph $\Gamma_{g}$ has zero length.

We prove this theorem by a sequence of lemmas. Our first lemma shows that our assumptions imply that all 2-cycles in $\Gamma_{g}$ have zero length. ${ }^{8}$ Note that all 2-cycles in $\Gamma_{g}$ have zero length if and only if $\ell$ is antisymmetric. That is, $\ell(a, b)=-\ell(b, a)$ for all $a, b \in A$.

Lemma 1. Under the assumptions of Theorem 8, any 2-cycle in the allocation graph $\Gamma_{g}$ has zero length.

Proof. Consider any $a, b \in A$. We first prove that $\ell(a, b)+\ell(b, a)=0$ when $U_{a}$ and $U_{b}$ are both finite. On the contrary, suppose that the sum of these lengths differs from zero, which by the 2-cycle nonnegativity condition implies that there exists an arbitrarily small $\delta>0$ such that

$$
\ell(a, b)+\ell(b, a) \geq \delta .
$$

We assume that

$$
U_{a}-\ell(b, a) \leq U_{b}
$$

This assumption is without loss of generality because if (13) does not hold, then the 2-cycle nonnegativity condition implies that $U_{b}-\ell(a, b) \leq U_{b}+$ $\ell(b, a)<U_{a}$, and we can reverse the roles of $a$ and $b$.

Consider any $v$ in the interior of $Q_{a} \cap \mathcal{V}$. By Theorem 6, the characteristic type $t^{v}$ is in $R_{a}$. Because $v$ is in the interior of $Q_{a} \cap \mathcal{V}$,

$$
v_{a}-v_{b}>\ell(b, a)
$$

and $v_{a}<U_{a}$. The latter inequality and (13) imply that

$$
v_{a}-\ell(b, a)<U_{b} .
$$

Define the valuation type $\tilde{v}$ by setting

$$
\tilde{v}_{a}=v_{a}+\varepsilon, \tilde{v}_{b}=v_{a}-\ell(b, a)+2 \varepsilon, \text { and } \tilde{v}_{c}=v_{c}, \forall c \in A \backslash\{a, b\},
$$

\footnotetext{
${ }^{8}$ For the special case in which $\mathcal{V}$ is all of $\mathbb{R}^{m}$, this lemma has also been established
} by Lavi, Mu'alem, and Nisan (2009, Claim 8). 


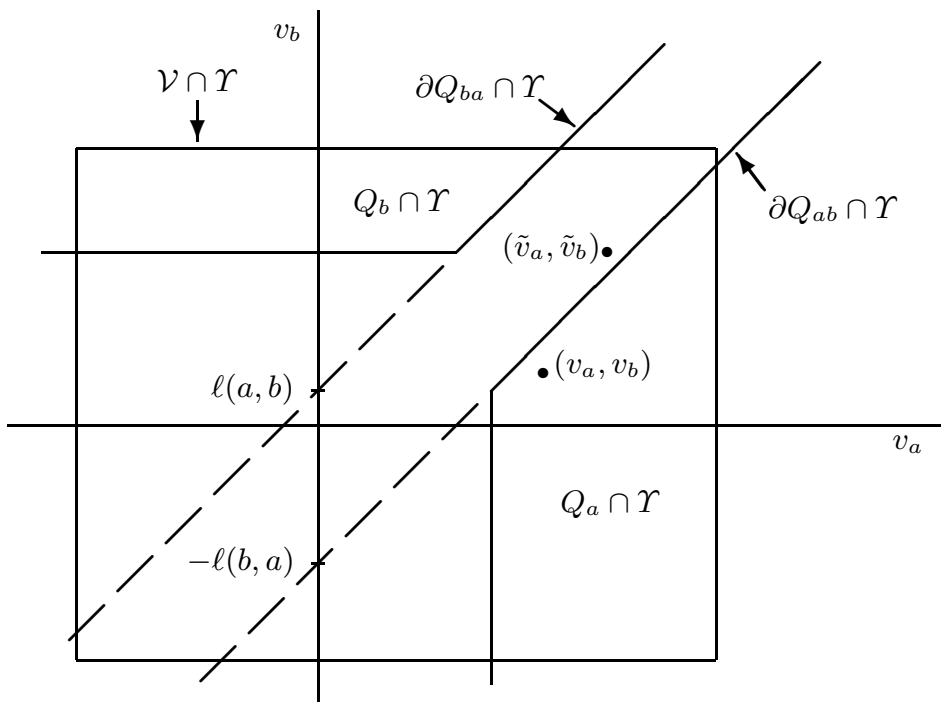

Fig. 3. Illustration of the Proof of Lemma 1.

where $\varepsilon>0$ is chosen to be sufficiently close to 0 so that both $\tilde{v}$ is in $\mathcal{V}$ and $\delta>\varepsilon$. Because $v_{a}<U_{a},(15)$ ensures that such a $\tilde{v}$ exists. Note that (14) and (16) imply that $\tilde{v}_{b}>v_{b}$.

It follows from (16) that

$$
\tilde{v}_{a}-\tilde{v}_{b}=\ell(b, a)-\varepsilon<\ell(b, a) .
$$

Hence, by Theorem 6 , the characteristic type $t^{\tilde{v}}$ cannot be in $R_{a}$. From (12), (16), and the assumption that $\delta>\varepsilon$, we have

$$
\tilde{v}_{b}-\tilde{v}_{a}=\varepsilon-\ell(b, a) \leq \varepsilon+\ell(a, b)-\delta<\ell(a, b),
$$

and so by Theorem $6, t^{\tilde{v}}$ cannot be in $R_{b}$. By construction, $\tilde{v} \geq v, \tilde{v}_{a}>v_{a}$, and $\tilde{v}_{c}=v_{c}$ for all $c \in A \backslash\{a, b\}$. Because $t^{v} \in R_{a}$, Theorem 7 implies that $t^{\tilde{v}}$ is not in $R_{c}$ for any $c \in A \backslash\{a, b\}$. We have shown that $g$ does not assign any outcome to $t^{\tilde{v}}$, which is impossible. Therefore, $\ell(a, b)+\ell(b, a)=0$.

If $U_{b}=\infty$, we do not need to assume (13) in order for (15) to hold, which is all that is needed in order for $\tilde{v}$ to be in $\mathcal{V} .^{9}$ If $U_{b}$ is finite, but $U_{a}=\infty$, an analogous argument with the roles of $a$ and $b$ reversed ensures that the requisite $\tilde{v}$ exists.

The proof of Lemma 1 is illustrated in Figure 3 for the case in which $\mathcal{V}$ is compact. In this diagram,

$$
\Upsilon=\left\{\hat{v} \in \mathbb{R}^{m} \mid \hat{v}_{c}=v_{c}, \forall c \in A \backslash\{a, b\}\right\},
$$

${ }^{9}$ If $U_{b}=\infty,(13)$ is satisfied if $U_{a}$ is finite. 
for some fixed values of $v_{c}$ for $c \in A \backslash\{a, b\}$ that will be specified later. The sets $\partial Q_{a b} \cap \Upsilon, \partial Q_{b a} \cap \Upsilon$, and $\mathcal{V} \cap \Upsilon$ are all independent of the valuations chosen for the outcomes other than $a$ and $b$. The upward sloping parts of $\partial Q_{a} \cap \Upsilon$ and $\partial Q_{b} \cap \Upsilon$ are contained in $\partial Q_{a b} \cap \Upsilon$ and $\partial Q_{b a} \cap \Upsilon$, respectively. By way of contradiction, we suppose that $\ell(a, b)+\ell(b, a)>0$, which, by the 2-cycle nonnegativity condition, implies that $\partial Q_{b a} \cap \Upsilon$ lies above $\partial Q_{a b} \cap \Upsilon$ when $v_{a}$ is plotted on the horizontal axis and $v_{b}$ is plotted on the vertical axis. Our interiority assumption ensures that (i) $\partial Q_{a b} \cap \Upsilon$ intersects the right-hand boundary of $\mathcal{V} \cap \Upsilon$ or (ii) $\partial Q_{b a} \cap \Upsilon$ intersects the upper boundary of $\mathcal{V} \cap \Upsilon$. Without loss of generality, we consider case (i).

We choose $v$ so that it is in the interior of $Q_{a} \cap \mathcal{V}$, which is possible by our interiority assumption. The valuation vector $\tilde{v}$ differs from $v$ only in the valuations of outcomes $a$ and $b$. It is chosen so that $\tilde{v}_{a}>v_{a}$ and $\tilde{v}_{b}>v_{b}$, and so that $\left(\tilde{v}_{a}, \tilde{v}_{b}\right)$ is not in either $Q_{a} \cap \Upsilon$ or $Q_{b} \cap \Upsilon$, as shown in the diagram. Because $v$ is in the interior of $Q_{a}$, by Theorem 6 , the characteristic type $t^{v}$ associated with $v$ is allocated $a$. Because $\tilde{v}$ is in neither $Q_{a}$ nor $Q_{b}$, the same theorem implies that the characteristic type $t^{\tilde{v}}$ associated with $\tilde{v}$ cannot be allocated either $a$ or $b$. In moving from $v$ to $\tilde{v}$, the valuations of $a$ and $b$ have increased with no change in the valuations of the other outcomes. Hence, by Theorem 7 , no outcome other than $a$ or $b$ can be allocated to $t^{\tilde{v}}$. We now have no outcome allocated to $t^{\tilde{v}}$, which is impossible, and so we conclude that $\ell(a, b)+\ell(b, a)=0$. Note that $-\ell(b, a)$ is the vertical intercept of $\partial Q_{a b} \cap \Upsilon$ and $\ell(a, b)$ is the vertical intercept of $\partial Q_{b a} \cap \Upsilon$. When these two values concide, then so do $\partial Q_{a b} \cap \Upsilon$ and $\partial Q_{b a} \cap \Upsilon$.

If there are only two outcomes, say $a_{1}$ and $a_{2}$, only the 2-cycle nonnegativity condition and convexity of $\mathcal{V}$ are needed to conclude that $\ell\left(a_{1}, a_{2}\right)+$ $\ell\left(a_{2}, a_{1}\right)=0$. This can be seen using Figure 1. Because $\mathcal{V}$ must intersect both $Q_{a_{1}}$ and $Q_{a_{2}}$, if, as shown in this diagram, $\ell\left(a_{1}, a_{2}\right)+\ell\left(a_{2}, a_{1}\right)>0$, then $\mathcal{V}$ must contain valuation vectors that are in neither of the two difference sets when $\mathcal{V}$ is convex. But then some types are not assigned any outcome, which is impossible.

We now show that if the length of every 2-cycle is zero and the length of every 3 -cycle is nonnegative, then all $k$-cycles have zero length.

Lemma 2. If all 2-cycles in the allocation graph $\Gamma_{g}$ have zero length and all 3-cycles in $\Gamma_{g}$ have nonnegative length, then for every integer $k \geq 2$, any $k$-cycle in $\Gamma_{g}$ has zero length.

Proof. By Lemma 1, any 2-cycle has zero length.

Consider any 3 -cycle $\left(a_{1}, a_{2}\right),\left(a_{2}, a_{3}\right),\left(a_{3}, a_{1}\right)$. Because all 3 -cycles have nonnegative length,

$$
\ell\left(a_{1}, a_{2}\right)+\ell\left(a_{2}, a_{3}\right)+\ell\left(a_{3}, a_{1}\right) \geq 0 .
$$

Because all 2-cycles have zero length, this inequality is equivalent to

$$
-\ell\left(a_{2}, a_{1}\right)-\ell\left(a_{3}, a_{2}\right)-\ell\left(a_{1}, a_{3}\right) \geq 0,
$$




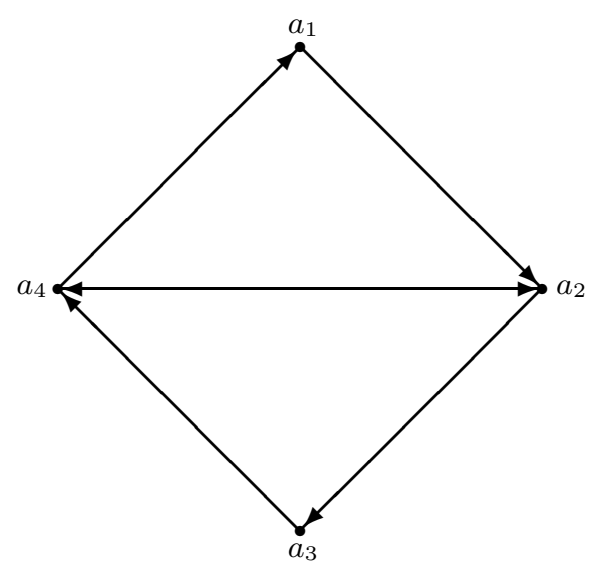

Fig. 4. Inserting a 2-cycle into a 4-cycle.

or, equivalently,

$$
\ell\left(a_{1}, a_{3}\right)+\ell\left(a_{3}, a_{2}\right)+\ell\left(a_{2}, a_{1}\right) \leq 0 .
$$

Because all 3-cycles have nonnegative length, the preceding inequality implies that the 3 -cycle $\left(a_{1}, a_{3}\right),\left(a_{3}, a_{2}\right),\left(a_{2}, a_{1}\right)$ has zero length, which implies that the original 3-cycle $\left(a_{1}, a_{2}\right),\left(a_{2}, a_{3}\right),\left(a_{3}, a_{1}\right)$ also has zero length.

Induction is used to complete the proof. Consider any integer $k \geq 4$ and suppose that any $(k-1)$-cycle has zero length. Consider any $k$-cycle $\left(a_{1}, a_{2}\right), \ldots,\left(a_{k-1}, a_{k}\right),\left(a_{k}, a_{1}\right)$. We now insert the 2 -cycle $\left(a_{k-1}, a_{1}\right),\left(a_{1}, a_{k-1}\right)$ before the arc $\left(a_{k-1}, a_{k}\right)$. This construction is illustrated in Figure 4 for the case in which $k=4$. The inserted 2-cycle has length zero. Thus,

$$
\begin{aligned}
\ell\left(a_{1}, a_{2}\right)+\cdots+\ell\left(a_{k-1}, a_{k}\right)+ & \ell\left(a_{k}, a_{1}\right) \\
=\left[\ell\left(a_{1}, a_{2}\right)+\cdots+\right. & \left.\ell\left(a_{k-2}, a_{k-1}\right)+\ell\left(a_{k-1}, a_{1}\right)\right] \\
& +\left[\ell\left(a_{1}, a_{k-1}\right)+\ell\left(a_{k-1}, a_{k}\right)+\ell\left(a_{k}, a_{1}\right)\right] .
\end{aligned}
$$

The first (resp. second) term in square brackets on the right-hand side of this equation is the length of a $(k-1)$-cycle (resp. 3-cycle). Both of these lengths are zero. Hence, the length of the original $k$-cycle is also zero.

To complete the proof of Theorem 8, it remains to show that the length of any 3-cycle is nonnegative.

Lemma 3. Under the assumptions of Theorem 8, any 3-cycle in the allocation graph $\Gamma_{g}$ has nonnegative length.

Proof. Consider any distinct $a, b, c \in A$. We first consider the case in which $L_{d} \neq-\infty$ for all $d \in A \backslash\{c\}$. Let 


$$
v_{c}^{*}=\max _{d \in A \backslash\{c\}}\left[L_{d}+\ell(d, c)\right]
$$

and consider any

$$
\bar{d} \in \arg \max _{d \in A \backslash\{c\}}\left[L_{d}+\ell(d, c)\right]
$$

Because the 2-cycle nonnegativity condition is satisfied, $\ell(d, c)$ is finite for all $d \in A \backslash\{c\}$. Thus, our assumptions ensure that such a $\bar{d}$ exists and that $v_{c}^{*}$ is finite. Note that for any $\bar{v}_{c}$ with $\bar{v}_{c}>v_{c}^{*}$, there exist $\bar{v}_{d}$ for all $d \in A \backslash\{c\}$ arbitrarily close to $L_{d}$ such that $\bar{v} \in Q_{c}^{\circ}$ and, hence by Theorem 6 , that $t^{\bar{v}} \in R_{c}$ if $\bar{v} \in \mathcal{V}$. ${ }^{10}$ We must have $v_{c}^{*}<U_{c}$, otherwise $Q_{c} \cap \mathcal{V}$ would have an empty interior. Furthermore, $L_{c}<v_{c}^{*}$, otherwise $t^{v} \in R_{c}$ for all $v$ with $v_{c}>v_{c}^{*}$, which implies that $Q_{d} \cap \mathcal{V}$ would have an empty interior for all $d \in A \backslash\{c\}$. Thus, $L_{c}<v_{c}^{*}<U_{c}$.

We now show that

$$
U_{a}-v_{c}^{*}>\ell(c, a) \text { and } U_{b}-v_{c}^{*}>\ell(c, b) .
$$

If the first inequality in (19) is violated, we have $U_{a}-v_{c}^{*} \leq \ell(c, a)$. Because $Q_{a} \cap \mathcal{V}$ has a nonempty interior, we must have

$$
U_{a}-L_{d}>\ell(d, a), \quad \forall d \in A \backslash\{a\} .
$$

Thus, by choosing $\varepsilon>0$ sufficiently small, there exists a valuation type $\tilde{v} \in \mathcal{V}$ defined by setting $\tilde{v}_{a}=U_{a}-\varepsilon, \tilde{v}_{c}=v_{c}^{*}-\varepsilon / 2$, and $\tilde{v}_{d}=L_{d}+\varepsilon$ for all $d \in A \backslash\{a, c\}$ such that $U_{a}-L_{d}>\ell(d, a)+2 \varepsilon$ for all $d \in A \backslash\{a, c\}$. However, we cannot assign any outcome in $A$ to valuation type $\tilde{v}$ because

$$
\begin{gathered}
\tilde{v}_{c}-\tilde{v}_{\bar{d}}=v_{c}^{*}-\varepsilon / 2-\left[L_{\bar{d}}+\varepsilon\right]<\ell(\bar{d}, c), \\
\tilde{v}_{a}-\tilde{v}_{c}=U_{a}-\varepsilon-\left[v_{c}^{*}-\varepsilon / 2\right]=U_{a}-v_{c}^{*}-\varepsilon / 2<U_{a}-v_{c}^{*} \leq \ell(c, a),
\end{gathered}
$$

and

$$
\begin{aligned}
\tilde{v}_{d}-\tilde{v}_{a}=L_{d}+\varepsilon-\left[U_{a}-\varepsilon\right] & =L_{d}-U_{a}+2 \varepsilon \\
& <-\ell(d, a)=\ell(a, d), \quad \forall d \in A \backslash\{a, c\},
\end{aligned}
$$

where the last inequality in (21) follows from (17), the last inequality in (22) holds by supposition, the inequality in (23) follows from (20), and the last equality in (23) follows because all 2-cycles have zero length. However, $\tilde{v}$ must be assigned an outcome in $A$, so this contradiction shows that $U_{a}-v_{c}^{*}>\ell(c, a)$. Similarly, we must have $U_{b}-v_{c}^{*}>\ell(c, b)$.

Contrary to what we want to show, now suppose that $\ell(a, b)+\ell(b, c)+$ $\ell(c, a)<0$. Let $\hat{v}$ be defined by setting

$$
\hat{v}_{a}=v_{c}^{*}-\ell(a, c)+2 \delta,
$$

\left.${\overline{10} \text { If } L_{d} \in\left\langle L_{d}\right.}_{,} U_{d}\right\rangle, \bar{v}_{d}$ can be chosen to be $L_{d}$. 


$$
\begin{gathered}
\hat{v}_{b}=v_{c}^{*}-\ell(b, c)+\xi, \\
\hat{v}_{c}=v_{c}^{*}+\delta
\end{gathered}
$$

and

$$
\hat{v}_{d}=L_{d}+\delta / 2, \quad \forall d \in A \backslash\{a, b, c\} .
$$

Because $L_{c}<v_{c}^{*}<U_{c}$, for $\delta>0$ sufficiently small, (26) and (27) imply that $L_{c}<\hat{v}_{c}<U_{c}$ and $L_{d}<\hat{v}_{d}<U_{d}$ for all $d \in A \backslash\{a, b, c\}$. Using (17), for $\delta>0$ and $\xi>0$ sufficiently small, it follows from (24) and (25) that $L_{a}<\hat{v}_{a}$ and $L_{b}<\hat{v}_{b}$. Because all 2-cycles have zero length, (24) and (25) also imply that $\hat{v}_{a}=v_{c}^{*}+\ell(c, a)+2 \delta$ and $\hat{v}_{b}=v_{c}^{*}+\ell(c, b)+\xi$. For $\delta>0$ and $\xi>0$ sufficiently small, it then follows from (19) that $\hat{v}_{a}<U_{a}$ and $\hat{v}_{b}<U_{b}$. Hence, by choosing $\delta>0$ and $\xi>0$ sufficiently small with $\delta>\xi$, it follows that $\hat{v} \in \mathcal{V}$ and

$$
\ell(a, b)+\ell(b, c)+\ell(c, a)+2 \delta-\xi<0 .
$$

We have

$\hat{v}_{a}-\hat{v}_{b}=\ell(b, c)-\ell(a, c)+2 \delta-\xi=\ell(b, c)+\ell(c, a)+2 \delta-\xi<-\ell(a, b)=\ell(b, a)$,

where the first equality follows from (24) and (25), the other two equalities follow because 2-cycles have zero length, and the inequality follows from (28). Thus, $a$ cannot be chosen when $v=\hat{v}$.

Because 2-cycles have zero length and $\delta>\xi$, it follows from (25) and (26) that

$$
\hat{v}_{b}-\hat{v}_{c}=-\ell(b, c)-\delta+\xi<-\ell(b, c)=\ell(c, b) .
$$

Thus, $b$ cannot be chosen when $v=\hat{v}$.

By (24) and (26),

$$
\hat{v}_{c}-\hat{v}_{a}=\ell(a, c)-\delta<\ell(a, c) .
$$

Thus, $c$ cannot be chosen when $v=\hat{v}$.

Finally, because 2-cycles have zero length, (17), (26), and (27) imply that

$$
\hat{v}_{d}-\hat{v}_{c}=L_{d}-v_{c}^{*}-\delta / 2<L_{d}-v_{c}^{*} \leq-\ell(d, c)=\ell(c, d), \quad \forall d \in A \backslash\{a, b, c\} .
$$

Thus, no $d \in A \backslash\{a, b, c\}$ can be chosen when $v=\hat{v}$.

We have shown that no outcome in $A$ can be chosen when $v=\hat{v}$, which is impossible. Thus, our supposition that $\ell(a, b)+\ell(b, c)+\ell(c, a)<0$ is false. Hence, $\ell(a, b)+\ell(b, c)+\ell(c, a) \geq 0$, which completes the proof when $L_{d} \neq-\infty$ for all $d \in A \backslash\{c\}$.

If some, but not all, $d \in A \backslash\{c\}$ have $L_{d}=-\infty$, the argument proceeds as above with a finite value $\bar{L}_{d}$ used instead of $L_{d}$ for all $d \in A \backslash\{c\}$ for which $L_{d}=-\infty$, where $\bar{L}_{d}$ is chosen to be sufficiently small so that $\bar{d}$ still solves (18) and (20) still holds. If $L_{d}=-\infty$ for all $d \in A \backslash\{c\}$, we then replace $L_{d}$ with a finite value $\bar{L}_{d}$ for all $d \in A \backslash\{c\}$, with $\bar{L}_{d}$ chosen so that (20) still holds and $v_{c}^{*}<U_{c}$. The proof then proceeds as above. 


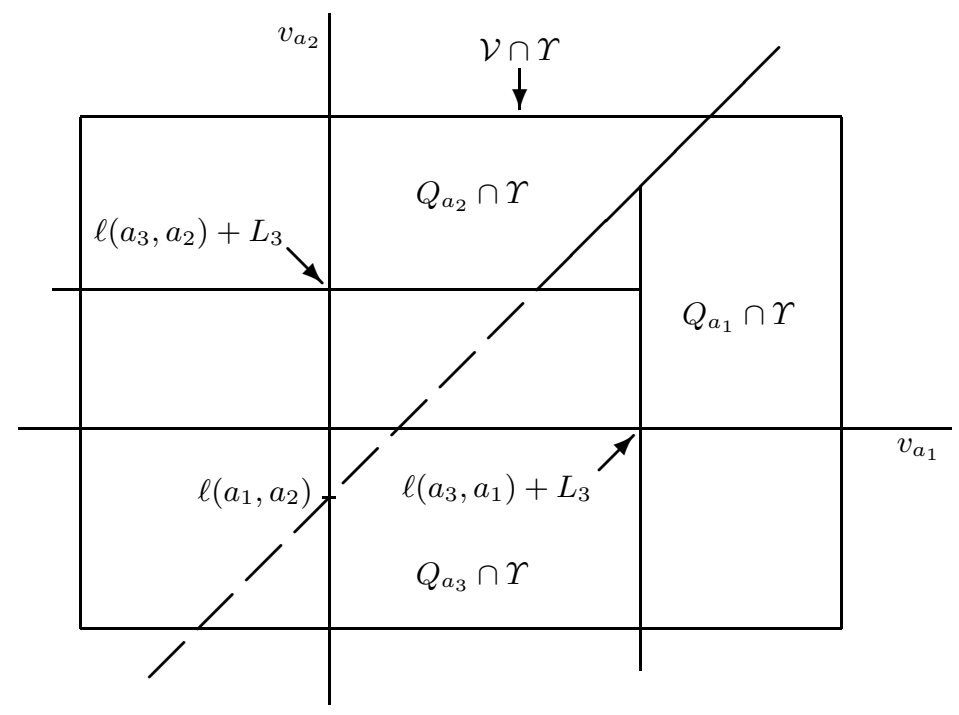

Fig. 5. 3-cycles with nonzero length.

The conclusion that the lengths of 3-cycles are zero follows from geometric properties of the difference sets that can be most easily seen when there are only three outcomes, say $a_{1}, a_{2}$, and $a_{3}$, and the valuation type space $\mathcal{V}$ is compact. Fix $v_{3}$ at its lowest value $L_{3}$ and let

$$
\Upsilon=\left\{v \in \mathbb{R}^{3} \mid v_{3}=L_{3}\right\}
$$

Recall that if we increase $v_{3}$, the left-hand boundary of $Q_{a_{1}}$ moves to the right and the lower boundary of $Q_{a_{2}}$ moves up. Thus, our interiority assumption implies that if we restrict attention to valuation vectors in $\Upsilon$, then the vertical part of the boundary of $Q_{a_{1}} \cap \Upsilon$ must lie to the left of the right-hand boundary of $\mathcal{V} \cap \Upsilon$ and the horizontal part of the boundary of $Q_{a_{2}} \cap \Upsilon$ must lie below the upper boundary of $\mathcal{V} \cap \Upsilon$, as illustrated in Figure 5. Because all 2-cycles have zero length, in $\Upsilon$, the upward sloping parts of the boundaries of $Q_{a_{1}}$ and $Q_{a_{2}}$ lie on a common line with slope equal to 1 , the vertical parts of the boundaries of $Q_{a_{1}}$ and $Q_{a_{3}}$ lie on a common vertical line, and the horizontal parts of the boundaries of $Q_{a_{2}}$ and $Q_{a_{3}}$ lie on a common horizontal line.

Suppose that the kinks on the boundaries of $Q_{a_{1}} \cap \Upsilon$ and $Q_{a_{2}} \cap \Upsilon$ do not coincide. Without loss of generality, we can suppose that the kink point for $Q_{a_{1}} \cap \Upsilon$ lies up and to the right of the kink point for $Q_{a_{2}} \cap \Upsilon$. As can be seen from the diagram, there is a triangular region in $\Upsilon$ whose interior is not in any of the three difference sets. Furthermore, either (i) the interior of this triangular region has a nonempty intersection with $\mathcal{V}$ or (ii) the interior of this triangular region lies below the lower horizontal boundary of $\mathcal{V} \cap \Upsilon$. In the latter case, we increase the value of $v_{3}$ until the interior of this triangular 


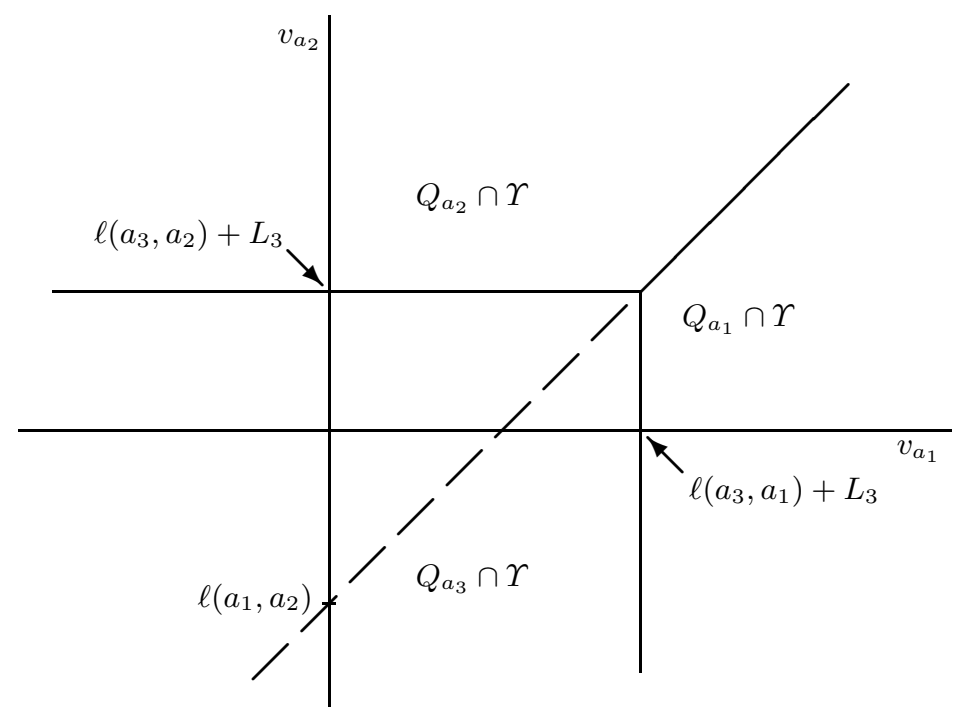

Fig. 6. 3-cycles with zero length.

region intersects with the new cross section of $\mathcal{V}$ obtained by increasing $v_{3}$ and define $\Upsilon$ using this new value of $v_{3}$. Because $Q_{a_{3}}^{\circ} \cap \mathcal{V} \neq \varnothing$, such a value of $v_{3}$ must exist. In Figure 5, all of the triangular region lies in $\mathcal{V} \cap \Upsilon$, but this need not be the case. Moreover, it is possible for $Q_{a_{3}} \cap \mathcal{V} \cap \Upsilon$ to be empty. By Theorem 6, it now follows that the characteristic types that correspond to valuation types in the intersection of the interior of the triangular region and $\mathcal{V}$ are not allocated any outcome, which is impossible. Thus, the kink points on the boundaries of $Q_{a_{1}} \cap \Upsilon$ and $Q_{a_{2}} \cap \Upsilon$ coincide, which implies that this common point is also the kink point on the boundary of $Q_{a_{3}} \cap \Upsilon$.

We now have a situation like that depicted in Figure $6 .^{11}$ Because the common boundary of $Q_{a_{1}} \cap \Upsilon$ and $Q_{a_{2}} \cap \Upsilon$ has a slope equal to 1 ,

$$
\ell\left(a_{3}, a_{2}\right)+L_{3}=\ell\left(a_{1}, a_{2}\right)+\ell\left(a_{3}, a_{1}\right)+L_{3} .
$$

Because all 2-cycles have zero length, $\ell$ is antisymmetric. Hence, (29) is equivalent to

$$
\ell\left(a_{1}, a_{2}\right)+\ell\left(a_{2}, a_{3}\right)+\ell\left(a_{3}, a_{1}\right)=0 .
$$

That is, the 3 -cycle $\left(a_{1}, a_{2}\right),\left(a_{2}, a_{3}\right),\left(a_{3}, a_{1}\right)$ has zero length. The antisymmetry of $\ell$ then implies that the only other 3-cycle, $\left(a_{1}, a_{3}\right),\left(a_{3}, a_{2}\right),\left(a_{2}, a_{1}\right)$, also has zero length. Note that for the situation shown in Figure 5 (which we have shown to be inconsistent with our assumptions), the right-hand side of

${ }^{11}$ If case (ii) in the preceding paragraph applies, then $L_{3}$ is replaced with the value of $v_{3}$ used to ensure that the interior of the triangular region contains valuation vectors in $\mathcal{V}$. 
(29) is larger than the left-hand side, from which it follows that the length of the 3 -cycle $\left(a_{1}, a_{2}\right),\left(a_{2}, a_{3}\right),\left(a_{3}, a_{1}\right)$ is positive and the length of the 3 -cycle $\left(a_{1}, a_{3}\right),\left(a_{3}, a_{2}\right),\left(a_{2}, a_{1}\right)$ is negative.

If $\mathcal{V}$ is a convex product space and our interiority assumption is satisfied, it follows from the Rockafellar-Rochet Theorem and Theorem 8 that a necessary condition for dominant strategy implementation of the allocation function $g$ is that all 2-cycles in the allocation graph $\Gamma_{g}$ have zero length. By Theorem 4 , if all of these 2-cycles have zero length, then $g$ is dominant strategy implementable. Thus, given our structural assumptions, we have identified a new necessary and sufficient condition for dominant strategy implementability of $g$ : all 2-cycles in the allocation graph $\Gamma_{g}$ have zero length. We combine this observation with the necessary and sufficient conditions in the RockafellarRochet and Saks-Yu Theorems in the following equivalence theorem.

Theorem 9. If the valuation type space $\mathcal{V}$ is a convex product space and $Q_{a} \cap \mathcal{V}$ has a nonempty interior for all $a \in A$, then the following conditions for the allocation function $\mathrm{g}: T \rightarrow A$ are equivalent:

(i) $g$ is dominant strategy implementable;

(ii) for every integer $k \geq 2$, any $k$-cycle in the allocation graph $\Gamma_{g}$ has nonnegative length;

(iii) any 2-cycle in the allocation graph $\Gamma_{g}$ has nonnegative length;

(iv) any 2-cycle in the allocation graph $\Gamma_{g}$ has zero length.

\section{Concluding Remarks}

We have shown that by requiring the valuation type space to be a convex product space and by adopting a mild domain regularity condition, the 2-cycle nonnegativity condition is sufficient for all $k$-cycles in an allocation graph to have zero length, not just to have nonnegative length, as is the case in Saks and Yu (2005). Furthermore, given our assumptions, a necessary and sufficient condition for dominant strategy implementability of the allocation function $g$ is that all 2-cycles in the allocation graph $\Gamma_{g}$ have zero length. As noted in Section 1, it follows from the analysis in Heydenreich, Müller, Uetz, and Vohra (2009) that this condition is necessary and sufficient for $g$ to satisfy the revenue equivalence property.

Our proof strategy utilizes the product space structure of a valuation type space in a number of steps in our proofs. It is an open question whether this structural condition can be relaxed. In particular, it is unknown whether our main theorem, Theorem 8 , continues to hold if the valuation type spaces are only required to be convex, as in Saks and Yu (2005). 


\section{Acknowledgements}

This article is based on John Weymark's plenary address to the conference, "New Developments in Social Choice and Welfare Theories: A Tribute to Maurice Salles," held at the Université de Caen. We have benefitted from the comments received on this occasion, as well as when this research was presented to the First UECE Lisbon Meetings on Game Theory and Applications, the Society for Social Choice and Welfare Conference in Moscow, and the Mathematics Colloquium at Vanderbilt University. We are grateful to Shurojit Chatterji, Paul Edelman, Mark Ellingham, Debasis Mishra, and Arunava Sen for discussions about this material.

\section{References}

Archer, A. and Kleinberg, R. (2008). Truthful germs are contagious: A local to global characterization of truthfulness. In Proceedings of the 9th ACM Conference on Electronic Commerce (EC'08), pages 21-30.

Ashlagi, I., Braverman, M., Hassidim, A., and Monderer, D. (2010). Monotonicity and implementation. Econometrica, 78, 1749-1772.

Berger, A., Müller, R., and Naeemi, S. H. (2009). Characterizing incentive compatibility for convex valuations. In M. Mavronicolas and V. G. Papadopoulou, editors, Algorithmic Game Theory, volume 5814 of Lecture Notes in Computer Science, pages 24-35. Springer-Verlag, Berlin.

Berger, A., Müller, R., and Naeemi, S. H. (2010). Path monotonicity and incentive compatibility. Research Memorandum No. RM/10/035, Maastricht Research School of Economics of Technology and Organization, Maastricht University.

Bikhchandani, S., Chatterji, S., Lavi, R., Mu'alem, A., Nisan, N., and Sen, A. (2006). Weak monotonicity characterizes deterministic dominant-strategy implementation. Econometrica, 74, 1109-1132.

Gui, H., Müller, R., and Vohra, R. V. (2004). Characterizing dominant strategy mechanisms with multi-dimensional types. Discussion Paper No. 1392, Center for Mathematical Studies in Economics and Management Science, Northwestern University.

Heydenreich, B., Müller, R., Uetz, M., and Vohra, R. V. (2009). Characterization of revenue equivalence. Econometrica, 77, 307-316.

Lavi, R., Mu'alem, A., and Nisan, N. (2009). Two simplified proofs for Roberts' theorem. Social Choice and Welfare, 32, 407-423.

Mishra, D. (2009). Monotonicity and incentive compatibility. Unpublished manuscript, Planning Unit, Indian Statistical Institute, Delhi Centre.

Myerson, R. B. (1981). Optimal auction design. Mathematics of Operations Research, 6, 58-73.

Roberts, K. (1979). The characterization of implementatable choice rules. In J.J. Laffont, editor, Aggregation and Revelation of Preferences, pages 321-348. North-Holland, Amsterdam.

Rochet, J.-C. (1987). A necessary and sufficient condition for rationalizability in a quasi-linear context. Journal of Mathematical Economics, 16, 191-200. 
Rockafellar, R. T. (1970). Convex Analysis. Princeton University Press, Princeton, NJ.

Saks, M. and Yu, L. (2005). Weak monotonicity suffices for truthfulness on convex domains. In Proceedings of the 6th ACM Conference on Electronic Commerce (EC'05), pages 286-293.

Vohra, R. V. (2011). Mechanism Design: A Linear Programming Approach. Cambridge University Press, Cambridge. 